\title{
Method for Identifying Micro-seismic P-Arrival by Time-frequency Analysis Using Intrinsic Time-Scale Decomposition
}

\author{
Ruihong ZHANG and Lihua ZHANG \\ Department of Civil Engineering, North China Institute of Science and Technology, \\ Sanhe-Hebei, China; e-mails: zhangruihongw@163.com, zhanglihua@ncist.edu.cn
}

\section{Abstract}

A method to identify the $P$-arrival of microseismic signals is proposed in this work, based on the algorithm of intrinsic timescale decomposition (ITD). Using the results of ITD decomposition of observed data, information of instantaneous amplitude and frequency can be determined. The improved ratio function of short-time average over long-time average and the information of instantaneous frequency are applied to the time-frequency-energy denoised signal for picking the $P$-arrival of the microseismic signal. We compared the proposed method with the wavelet transform method based on the denoised signal resulting from the best basis wavelet packet transform and the single-scale reconstruction of the wavelet transform. The comparison results showed that the new method is more effective and reliable for identifying $P$-arrivals of microseismic signals.

Key words: micro-earthquake, $P$-arrival, ITD, time-frequency analysis, denoising.

\section{INTRODUCTION}

Microseismic monitoring technology provides a good method for monitoring rock mass rupture during exploration and excavation. For early warning of rupture and to prevent damage caused by rock mass fracture, a rapid and re- 
liable monitoring method is needed to detect and accurately identify the arrival of the first microseismic signal.

As microseismic signals from mining activities usually propagate over short distances, the STA/LTA (the ratio of the Short Time Average over the Long Time Average) method of amplitude change is often used, with the first arrival of the $P$-wave taken as the trigger point. Although improved detection functions were developed (Allen 1978, 1982, McEvilly and Majer 1982, Baer and Kradolfer 1987, Earle and Shearer 1994), detecting the first arrival can result in a rough estimate because the accuracy of the STA/LTAbased method depends on the length of the time window and the predefined threshold (Ma 2008). Ye et al. (2008) used the maximum energy ratio of two time windows to detect the first arrival of microseismic waves. With this method, the results are still affected by the length of the time window and the shape of the waveform. In other studies that identified the first arrival of seismic waveforms (Zhang et al. 2003, Bai and Kennett 2000, Liu et al. 2013), large deviations exist between the real arrivals and the $P$-wave picking results (Dai and MacBeth 1995).

To overcome the difficulties mentioned above, in this paper, we introduce the method of intrinsic timescale decomposition (ITD) (Frei and Osorio 2007) and propose a highly accurate and efficient algorithm for identifying the first $P$-arrival of microseismic signals. Based on the time-frequencyenergy denoised instantaneous amplitude and frequency information that was obtained, an algorithm that accurately identifies the $P$-arrival is presented by using four parameters. Based on the instantaneous piece-wise constant amplitude and instantaneous frequency information, the thresholds for the identification scheme are easier to predefine and the adaptive calculation time window for the STA/LTA calculation can be derived. We apply this method to simulated and real data and show that the proposed method can accurately identify the first $P$-arrival of seismic signals, providing a feasible technological approach for detecting $P$-wave arrivals.

\section{INSTANTANEOUS TIME-FREQUENCY-ENERGY INFORMATION EXTRACTION AND DENOISING}

\subsection{Inherent timescale decomposition algorithm}

Given the signal $X_{t}$, the operator $L$ is defined whereby the baseline signal $L_{t}$ can be extracted from the signal $X_{t}$ so that the residual signal $H_{t}$ is a proper rotation. Hence, $X_{t}$ can be decomposed as $L_{t}$ and $H_{\mathrm{t}}$. Let $\left\{\tau_{k}, k=1,2, \ldots\right\}$ be the local extrema of $X_{t}, X_{k}$, and $L_{k}$ denoted $X\left(\tau_{k}\right)$ and $L\left(\tau_{k}\right)$, respectively, then the baseline signal $L_{t}$ on the interval $\left(\tau_{k}, \tau_{k+1}\right]$ between successive extrema is as follows (Frei and Osorio 2007): 


$$
L_{t}=L X_{t}=L_{k}+\left(\frac{L_{k+1}-L_{k}}{X_{k+1}-X_{k}}\right)\left(X_{t}-X_{k}\right), \quad \mathrm{t} \in\left(\tau_{k}, \tau_{k+1}\right],
$$

were

$$
L_{k+1}=\alpha\left[X_{k}+\left(\frac{\tau_{k+1}-\tau_{k}}{\tau_{k+2}-\tau_{k}}\right)\left(X_{k+2}-X_{k}\right)\right]+(1-\alpha) X_{k+1}
$$

and $\alpha$ is set as 0.5 . The residual signal $H_{t}$ is as follows:

$$
H_{t}=H X_{t}=X_{t}-L_{t} .
$$

To make the residual function monotonic between extrema, as required for proper rotations, the baseline is constructed as a linearly transformed contraction of the original signal. Once the original signal is decomposed, the decomposition can be applied to the baseline signal again. The decomposition procedure is iterated several times. Finally, the original signal can be decomposed into a series of proper rotation components of successively decreasing instantaneous frequencies at each subsequent level and a lowfrequency baseline signal. The decomposition iterative process is as follows (Frei and Osorio 2007):

$$
X_{t}=H X_{t}+L X_{t}=H X_{t}+(H+L) L X_{t}=\left(H \sum_{k=0}^{p-1} L^{k}+L^{p}\right) X_{t},
$$

where $H L^{k} X_{t}$ is the proper rotation of the $(k+1)$ th level, and $L^{p} X_{t}$ is the extracted lowest frequency baseline.

Through the operator $L$, the inherent information of the original signal $X_{t}$ (for example the extremum position) can be passed down to the baseline and the residual parts. The residual component $H_{t}$ and baseline $L_{t}$ can be monotonic on the interval $\left(\tau_{k}, \tau_{k+1}\right]$, the extrema $\left\{\tau_{k}, k \geq 1\right\}$ of $H_{t}$ coincide with the extrema of $X_{t}$, and the extrema $\left\{\tau_{k}, k \geq 1\right\}$ of $X_{t}$ are the extrema or inflection points for $L_{t}$. The initial decomposition of the signal on the interval $\left[0, \tau_{1}\right]$ can be extended to values of $t<0$ by reflections. Incomplete waves at the beginning and/or end of $H_{t}$, i.e., data prior to the first zero up-crossing or after the last zero up-crossing may be treated according to these definitions over the appropriate subinterval of $\left[t_{1}, t_{5}\right]$. $H_{t}$ is a proper rotation on the interval $\left[\tau_{1}, \tau_{N}\right]$ for any $N \geq 1$. The proof is as follows (ITD; Frei and Osorio 2007).

Assuming that $X_{t}$ has a local maximum at $\tau_{k}$ and adjacent local minima at $\tau_{k-1}$ and $\tau_{k+1}$, then $H_{\mathrm{t}}$ has a local maximum at $\tau_{k}$ and is monotonic on $\left[\tau_{k+1}, \tau_{k}\right]$ and on $\left[\tau_{k}, \tau_{k+1}\right]$ because:




where $\alpha_{K}=\left(L_{k}-L_{k-1}\right) /\left(X_{k}-X_{k-1}\right), \forall \mathrm{k}$.

If $X_{t}$ is monotonic on $\left[\tau_{k-1}, \tau_{k}\right]$ and on $\left[\tau_{k}, \tau_{k+1}\right]$, then $H_{t}$ is monotonic on $\left[\tau_{k-1}, \tau_{k}\right]$ and on $\left[\tau_{k}, \tau_{k+1}\right]$.

If $L_{k-1}>X_{k-1}, \quad L_{k+1}>X_{k+1}$, and $L_{k}<X_{k}$ then $H_{k-1}=X_{k-1}-L_{k-1}<0$, $H_{k}=X_{k}-L_{k}>0$, and $H_{k+1}=X_{k+1}-L_{k+1}<0$. Therefore, $H_{t}$ has a local maximum at $t=\tau_{k}$.

\subsection{The extraction of instantaneous information}

When a microseismic signal is decomposed into a series of intrinsic rotational components and a low-frequency baseline signal using ITD, the inherent instantaneous time-frequency-energy information of the input signal can be obtained by analyzing the morphology and other features of the waves. Specifically, for the intrinsic rotational components, based on the single waves and their monotonic segment between adjacent extrema, we get the instantaneous amplitude information, which is piece-wise constant, and the constant value is determined by the extremum values of the proper rotations between zero crossings from the signal $X_{t}$. For one full wave (i.e., the portion of the signal between successive zero up-crossings) at a time, the instantaneous amplitude information is as follows (Frei and Osorio 2007):

$$
A= \begin{cases}A_{1}, & \mathrm{t} \in\left[t_{1}, t_{3}\right) \\ -A_{2}, & \mathrm{t} \in\left[t_{3}, t_{5}\right),\end{cases}
$$

where $A_{1}>0$ and $A_{2}>0$ are the absolute amplitude of the positive and negative half-waves between successive zero up-crossings $t_{1}$ and $t_{5}$, respectively, and $t_{2}$ is the time of the positive half-wave maxima $\left(A_{1}\right), t_{3}$ is the time of the zero down-crossing, and $t_{4}$ is the time of the negative half-wave minima $\left(-A_{2}\right)$.

In accordance with the instantaneous amplitude, the instantaneous frequency is also piece-wise constant and can be computed between times of successive extrema of the ITD-generated proper rotation components, as follows (Frei and Osorio 2007):

$$
f_{t}=\left\{\begin{array}{ll}
\frac{0.5}{t_{3}-t_{1}}, & \mathrm{t} \in\left[t_{1}, t_{3}\right) \\
\frac{0.5}{t_{5}-t_{3}}, & \mathrm{t} \in\left[t_{3}, t_{5}\right)
\end{array} .\right.
$$

For simplicity, an alternative way to write the instantaneous period is

$$
\mathrm{T}_{t}=\left\{\begin{array}{ll}
2\left(t_{3}-t_{1}\right), & \mathrm{t} \in\left[t_{1}, t_{3}\right) \\
2\left(t_{5}-t_{3}\right), & \mathrm{t} \in\left[t_{3}, t_{5}\right)
\end{array} .\right.
$$


At the beginning of a proper rotation component, the incomplete waves, i.e., the data prior to the first zero up-crossing, or the incomplete waves at the end of a proper rotation component, i.e., the data after the last zero upcrossing, may be processed according to these definitions over the appropriate subinterval of $\left[t_{1}, t_{5}\right]$.

\subsection{ITD-based time-frequency-energy denoising}

The accuracy of the detection of the first arrival is dependent on the SNR of the recorded signals; therefore denoising is an important stage of the arrival detection process. It is necessary to reduce the interference noise of the microseismic signals as much as possible. As any slight noise, distortion, or phase shift on the first arrival can result in an incorrect time picking (Galiana-Merino et al. 2003), a denoising method based on ITD (Zhang et al. 2012) is more suitable for detecting the seismic signal because the abrupt change of the amplitude at the beginning of the first pulse is preserved. We therefore apply the ITD method to the microseismic signals.

To carry out ITD-based time-frequency-energy denoising, two steps are needed: (i) the instantaneous frequencies and instantaneous amplitudes are extracted for each of the ITD-generated proper rotation components of the original signal; and (ii) the denoised components are obtained by using the amplitude thresholds and period (frequency) thresholds.

The decomposition levels are dependent on the ratio of the average amplitude energy of the denoised components to the amplitude energy of the total denoised signal. Generally, we used the first several components containing the main frequency bands of a microseismic event. Ultimately, the time-frequency-energy denoised instantaneous amplitude signal and frequency characteristics were obtained for the first pulse detection.

Figure 1 illustrates the process of ITD-based time-frequency-energy denoising. First, a synthetic signal was generated at frequencies of 6,7 , and $10 \mathrm{~Hz}$ (Fig. 1a). The synthetic signal begins at $3 \mathrm{~s}$, using a sample frequency of $200 \mathrm{~Hz}$. The pure synthetic signal was contaminated with Gaussian noise so that the SNR is $10 \mathrm{~dB}$ (Fig. 1b). Figure 1c shows the original decomposed piece-wise constant instantaneous amplitude of the first four components, and Fig. 1d shows the original piece-wise constant instantaneous period of the first four components. Figure 1e shows the denoised instantaneous amplitude of the first four components, and Fig. 1f shows the filtered instantaneous period of the first four components. Using the ITD method, the leveldependent amplitude thresholds and frequency thresholds were applied according to the noise samples, which were several seconds ahead of the signal (Zhang et al. 2012); meanwhile the denoised instantaneous amplitudes were filtered by the high-frequency thresholds and the instantaneous period in- 

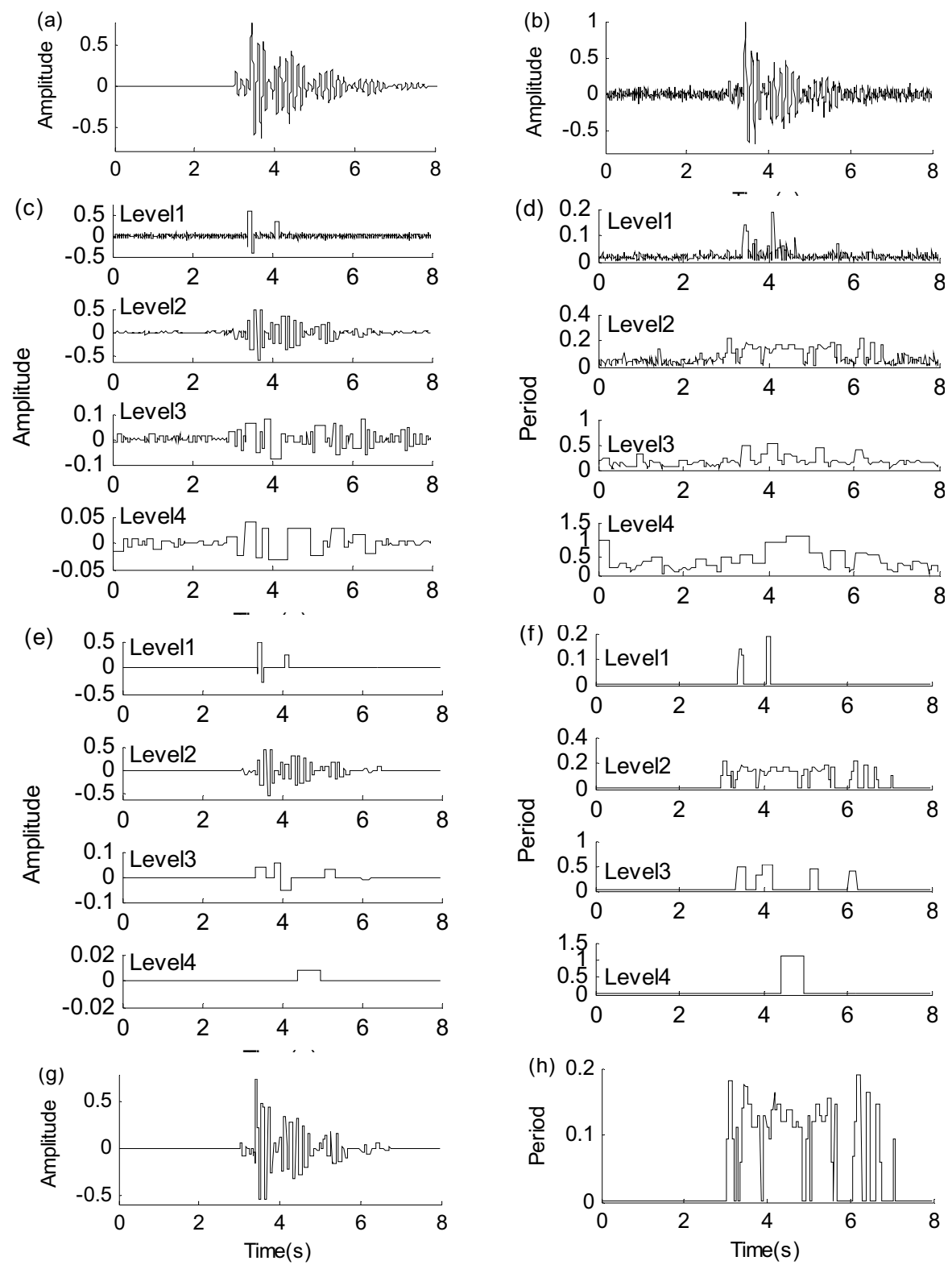

Fig. 1. Denoising process for a contaminated synthetic signal using the ITD method: (a) pure synthetic signal generated by several sine waves damped by an exponential, (b) synthetic signal contaminated by Gaussian noise, (c) the original piece-wise constant amplitude of the first four components, (d) the original piece-wise constant period of the first four components, (e) time-frequency-energy denoised instantaneous amplitude of the first four components, (f) filtered instantaneous period of the first four components, $(\mathrm{g})$ denoised amplitude from the first three amplitude components, and $(\mathrm{h})$ equivalent period calculated from the first two components. 
formation was also dependent on the denoised amplitude information. The extrema of the denoised discomposed components coincide with those of the original signal, and the instantaneous period components are in good agreement with the instantaneous amplitude components, demonstrating the advantage of the proposed method for first arrival detection.

Table 1 presents the average amplitude and frequency of the denoised first four components. The two maximum energy components of levels 2 and 1 account for $80 \%$ of the total energy. The energy from level 4 and subsequent levels comprises nearly $9 \%$ of the total energy, the frequency of level 3 is lower than the generated frequency, and further decomposition is not needed. The sum of the first three amplitude components that contain the components of the three maximum energy levels as the instantaneous amplitude information is reasonable (Fig. 1g). We use the equivalent period as the instantaneous frequency information (Fig. 1h). The equivalent period is the energy-weighted sum of the frequency components by the average amplitude ratio of the two maximum energy components. The equivalent average frequency of levels 2 and 1 is 6.89 according to this conversion which is a good estimate of the main frequency of 7.

Table 1

Average amplitude and frequency of the denoised components

\begin{tabular}{|c|c|c|c|}
\hline $\begin{array}{c}\text { Component } \\
\text { level }\end{array}$ & $\begin{array}{c}\text { Average frequency } \\
{[\mathrm{Hz}]}\end{array}$ & $\begin{array}{c}\text { Average } \\
\text { amplitude }\end{array}$ & $\begin{array}{c}\text { Amplitude ratio } \\
{[\%]}\end{array}$ \\
\hline 1 & 6.92 & 0.0098 & 11.2 \\
2 & 6.88 & 0.0602 & 69.0 \\
3 & 3.76 & 0.0095 & 10.9 \\
4 & 1.39 & 0.0033 & 3.8 \\
\hline
\end{tabular}

\section{THE PROPOSED ALGORITHM}

An algorithm for the first pulse identification was proposed based on the denoised piece-wise constant amplitude and the equivalent period (frequency) information. First, the thresholds of the instantaneous amplitude ratio (STA/BTA) and the instantaneous period (FSTA) were predefined. STA is the maximum amplitude of the half-wave that the detected point belongs to, BTA is the maximum amplitude of the wave that the previous point belongs to, and FSTA is the instantaneous period average of the half-wave that the detected point belongs to. When STA/BTA and FSTA exceed their predetermined thresholds, the average amplitude ratio (LTA/BTA) and the average period (FLTA) in the next period of time of the signal will be calculated based on the information of that period. LTA and FLTA are the long-term- 
average amplitude and the average period of the next consecutive period time related to the detected point, respectively. When LTA/BTA and FLTA are larger than their predetermined thresholds, this indicates that a microseismic event is occurring. The first two parameters ensure that the instantaneous amplitude and period are consistent with a microseismic event. The last two parameters can prevent a false detection caused by individual disturbance fluctuations. The detailed steps are as follows:

a The original signals are decomposed by ITD and the components of the first three or four levels are generated; the four components generally contain the three maximum energy levels and include the main frequency bands of the original signals.

- Instantaneous amplitude and period (frequency) information is extracted from the decomposition components. The instantaneous amplitude components are denoised and the period components are filtered by leveldependent amplitude thresholds and level-dependent period thresholds, respectively. The denoised instantaneous amplitude is filtered by the highfrequency threshold and the instantaneous period is also dependent on the denoised amplitude information; thus, the amplitude and period information are interdependent.

a The first several levels (generally three levels) of the denoised amplitude components are summed as the instantaneous amplitude information; these components contain the two maximum energy levels. The energyweighted period components of the two maximum energy levels are summed as the equivalent period information; the weighted coefficients may be determined by the average amplitude ratio of the two maximum energy levels (such as levels 1 and 2 in Table 1).

- STA/BTA and FSTA are detected for each point. When FSTA and STA/BTA are higher than the two predefined thresholds, the next step is followed.

- LTA and FLTA of the next consecutive period time connected with the detected point are calculated. The optimum time window for calculating LTA and FLTA depends on the period information. If LTA/BTA and FLTA are higher than the two thresholds then a phase arrival is affirmed and the first-arrival time is determined.

The first-arrival is defined as the first sample where all four parameters (STA/BTA, FSTA, LTA/BTA, and FLTA) exceed their thresholds. As the instantaneous piece-wise constant amplitude is determined by the extremum values between the zero crossings rather than by the transient amplitude, it is easy to predefine the amplitude threshold for the first-arrival detection because the abrupt extremum value is far larger than the low transient amplitude value at the beginning of a pulse. For a routine transient amplitude signal, because of the low transient amplitude at the beginning of the first 
pulse, the threshold is difficult to predetermine and often gives false picking results. Based on the period (frequency) information of the signal, the location of the next wave information can be determined; therefore, the adaptive time window can be obtained for the proposed algorithm.

It is worth mentioning that as the proposed scheme is applied to the previously denoised signal, there may be a number of samples preceding the first arrival that were reduced to zero or to a very low value, which could produce a low BTA value. Therefore, stability factors should be introduced when calculating the ratio-related values.

\section{MODELING RESULTS BY MATLAB}

To evaluate the proposed method, first-arrival identification was performed for the contaminated synthetic signal of Fig. 1b, first by the proposed method and then using the wavelet transform (WT) method, and the results were compared.

The first-arrival detection of the proposed method is illustrated in Fig. 2a. The equivalent periods were calculated from the instantaneous peri-
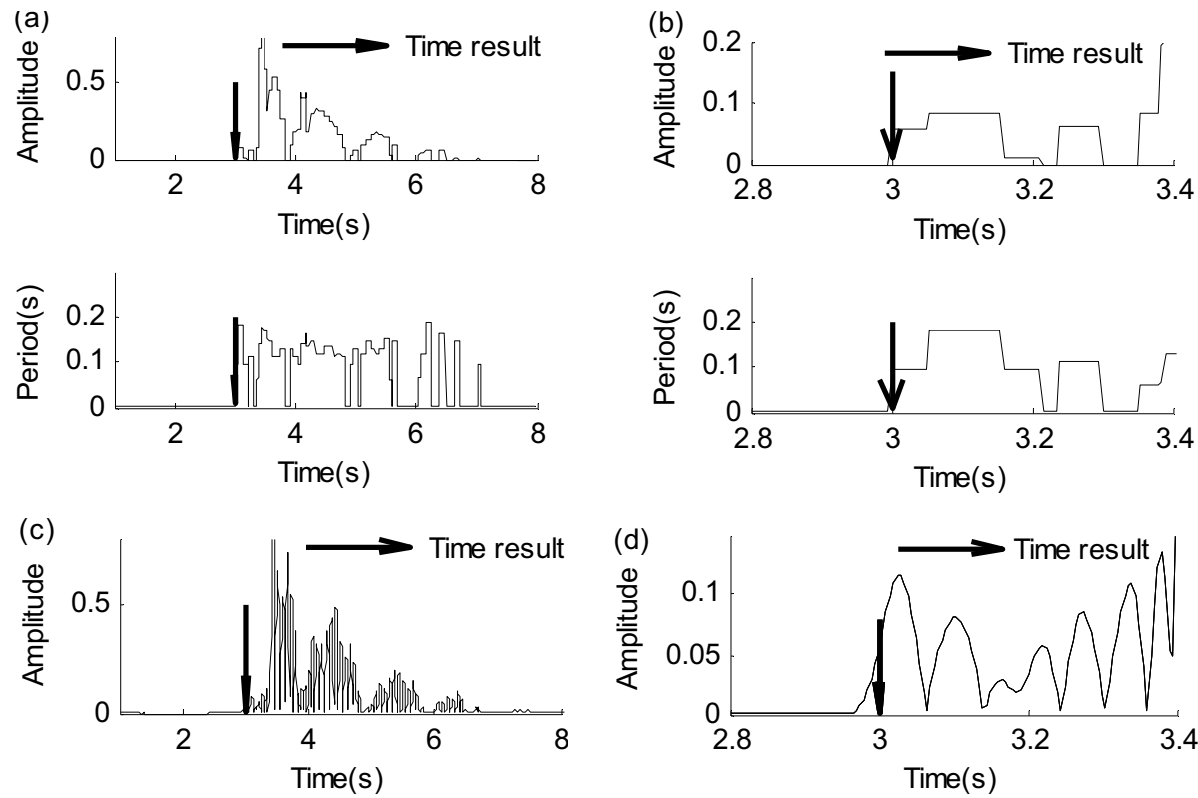

Fig. 2. First-arrival identification by two methods for the contaminated synthetic signal shown in Fig. 1b: (a) the absolute denoised amplitude and equivalent period used by the proposed method and the picking result, (b) the local magnified drawing near the picking results of Fig. 2a, (c) the normalized absolute reconstructed signal used by the WT method and the picking result, and (d) the local magnified drawing near the picking results of Fig. 2c. 
od components of levels 1 and 2; the energy weighted coefficients are the average amplitude ratios of the two maximum energy components. The instantaneous amplitude signals used the sum of the first two denoised amplitude components including the two maximum energy levels. The thresholds of FSTA and FLTA were estimated based on the equivalent period threshold for period filtering and the average equivalent period, respectively. The thresholds used for STA/BTA, LTA/BTA, FSTA, and FLTA were 1.5, 1.5, 0.03 , and 0.06 , respectively. The automatic first-arrival picking result was $3.000 \mathrm{~s}$, indicated by the vertical dashed line.

The WT method of first-arrival detection uses the normalized singlescale reconstructed signals by wavelet transformation of the denoised signals. The denoised signals were obtained through the optimum wavelet packet transform methods by using a node-dependent threshold of the noise (Zhang et al. 2011). The first-arrival times were detected by the parameter thresholds of STA/LTA and LTA. When the ratio of STA/LTA and LTA exceeded their predetermined thresholds, the first $P$-arrival time was identified (Galiana-Merino et al. 2007)

The Daubechies 8 orthogonal mother wavelet (Daubechies 1992) was employed, four levels of decomposition were used for the WT algorithm, and a normalized single-scale reconstructed signal of the 4th scale was obtained for the first-arrival detection. The normalized absolute single-scale reconstructed signal used by the WT method is shown in Fig. 2c. The calculation time windows used for the WT algorithm were: an STA window of 20 samples (the sampling rate of the signal was $5 \mathrm{~ms}$, equivalent to one period of a $10-\mathrm{Hz}$ pulse), an LTA window of 80 samples (four times the STA window length). The threshold of STA/LTA was 2.6, and that of LTA was 0.002 . The automatic picking result indicated by the long vertical dashed line is $3.000 \mathrm{~s}$.

Figure $2 b$ and $d$ shows a magnified view of Fig. $2 a$ and $c$ near the firstarrival picking results of the two methods, respectively. The picking point of each method is indicated by a vertical dashed line.

For the proposed method, the instantaneous amplitude and equivalent period of the original signal are obtained, and the denoised amplitude preserves the sudden change of amplitude at the beginning of the first pulse, as well as prevents the distortion and phase shift phenomenon (Fig. 2b). Moreover, the instantaneous equivalent period of the original signal that is consistent with its amplitude information provides an important evaluation criterion for the first pulse identification. This suggests that the proposed method provides better identification of the first pulse. The WT denoised waveform (Fig. 2d) is smooth; however, because of the inherent characteristic of the wavelet, distortion, and a phase-shift phenomenon occur in the denoised reconstruct- 
ed signal at the beginning of the first arrival; this can easily lead to an identification of the first arrival earlier than it actually was.

\section{APPLICATION EXAMPLES}

Two applications of the two methods are illustrated as follows.

Figure 3 shows the first-arrival picking results for manmade blasting signals of high SNR using the two methods mentioned above. The six original signals of the manmade blasting are shown in Fig. 3a. The equivalent periods used by the proposed method are presented in Fig. 3b. In Fig. 3c, the denoised amplitude information used by the proposed method and the denoised normalized single scale reconstructed signals used by the WT method are compared with the original signals.

For the first arrival detection by the proposed method, the first maximum energy level is level 2 and the second maximum energy level is level 3 for all signals. Therefore, the equivalent periods were all calculated from the instantaneous period components of levels 2 and 3; the instantaneous amplitude signals used the sum of the first three amplitude components including the two maximum energy levels. The first component of level 1 can be ignored because of its very low energy and high frequency. The identification thresholds used here for STA/BTA, LTA/STA, FSTA, and FLTA are 1.5, $1.5,0.002$, and 0.004 , respectively.

For the first arrival detection by the WT method, the threshold of STA/LTA was 2.6, and that of LTA was 0.001 . The main frequency band derived from the FFT of the original signals was $10-60 \mathrm{~Hz}$. The time windows for the calculation were: an STA window of 50 samples (the sampling rate of the signal is $0.2 \mathrm{~ms}$, equivalent to half a period of a $50-\mathrm{Hz}$ pulse), and an LTA window of 200 samples (four times the STA window length).

Table 2 shows the automatic picking results of the proposed method and the WT method and their errors compared with the manual picking results. As the original manmade blasting signals have a high SNR, the manual results have small errors compared with the real results. The difference between the automatic picking results and the manual results may be taken as the errors of the picking results.

The first-arrival picking results for low SNR microseismic signals by the two automatic methods are shown in Fig. 4. Figure 4a shows the waveforms of six microseismic signals recorded in a mine. The equivalent periods used by the proposed method are presented in Fig. 4b. The amplitude information used by the proposed method and the normalized reconstructed signals used by the WT method compared with the original signals near the picking results are shown in Fig. 4c.

In the proposed method, the two maximum energy levels are levels 2 and 3 for signals S1-S6 except of S4; the maximum energy level for S4 is 



(c) $\quad$ Original Amplitude $\ldots \ldots \ldots$ Amplitude by WT
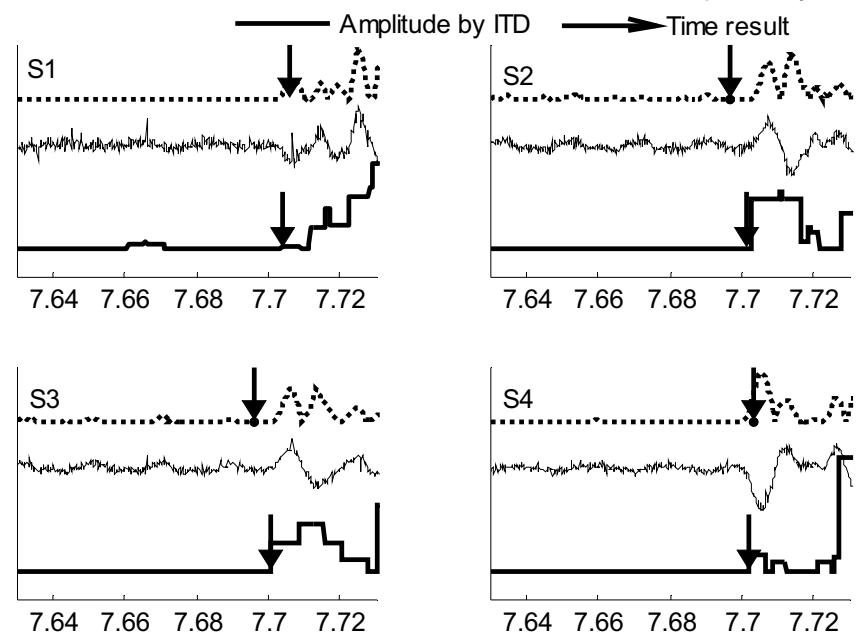

Fig. 3. First-arrival identification for six manmade blasting signals by two automatic methods: (a) the six original signals of manmade blasting, (b) period information used by the proposed method near the picking results, and (c) comparison of the amplitude used by the proposed meth-

od and the normalized single scale reconstructed signals used by the WT method with the original signals near the picking results. 
Picking results comparison of manmade blasting waves

\begin{tabular}{|c|c|c|r|r|r|}
\hline \multirow{2}{*}{$\begin{array}{c}\text { Signal } \\
\text { number }\end{array}$} & \multirow{2}{*}{$\begin{array}{c}\text { Manual } \\
\text { results }\end{array}$} & \multicolumn{2}{|c|}{ Proposed method } & \multicolumn{2}{c|}{ WT method } \\
\cline { 3 - 6 } & Results & Error* & Results & \multicolumn{1}{|c|}{ Error* } \\
\hline 1 & 7.7030 & 7.7038 & 0.0008 & 7.7062 & 0.0032 \\
2 & 7.7030 & 7.7014 & -0.0016 & 7.6970 & -0.0060 \\
3 & 7.7020 & 7.7008 & -0.0012 & 7.6962 & -0.0058 \\
4 & 7.7010 & 7.7020 & 0.0010 & 7.7032 & 0.0022 \\
5 & 7.7070 & 7.7048 & -0.0022 & 7.7106 & 0.0036 \\
6 & 7.7010 & 7.6998 & -0.0012 & 7.6960 & -0.0050 \\
\hline
\end{tabular}

${ }^{*}$ The error means the difference between the manual picking result and the automated methods result.

level 1. For consistency, the amplitude signals and the equivalent periods were calculated by using the first two maximum energy levels for all the signals. The thresholds for STA/BTA, LTA/STA, FSTA, and FLTA were 1.5, $1.5,0.001$, and 0.002 .

For the WT method, the threshold of STA/LTA was 2.6 and the threshold of LTA was 0.003 . The main frequency band of the original signals was $50-150 \mathrm{~Hz}$. The time windows for the calculation were: an STA window of 50 samples (signal sampling rate of $0.2 \mathrm{~ms}$, equivalent to a period of a $100-\mathrm{Hz}$ pulse), and an LTA window of 200 samples (four times the STA window length).

Table 3 shows the automatic picking results of the proposed method and the WT method and their errors compared with the manual picking results. As the microseismic signals have low SNR, the manual results are less reliable than those of the manmade blasting signals. The errors of the automatic picking results were also estimated from the difference between the automatic picking results and the manual results.

Comparing the results from Tables 2 and 3, the errors of the WT method were bigger than the errors of the proposed method in most cases, and were less than those of the proposed method in a few cases. For high SNR signals, the differences between the proposed method and the manual results are less than $0.00 \pm 0.002 \mathrm{~s}$ in most cases; for low SNR signals, the errors of the proposed method are within $0.00 \pm 0.003 \mathrm{~s}$ in most cases; if the discomposed components of level 4 were included in the amplitude and the period information for low SNR microseismic signals, the errors between the proposed method and the manual results would be less, especially for signal S6 in Fig. 4.

In fact, the WT denoised signals were roughly the same as the ITD denoised signals in most cases. For the high-SNR signals, the WT denoised 
(a) $\mathrm{S} 1$

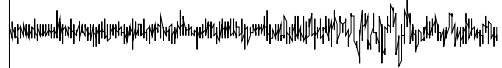

S2

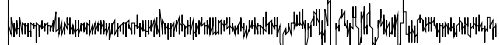

S3

$\frac{\text { 을 }}{\frac{3}{2}}$

S4

S5

s6

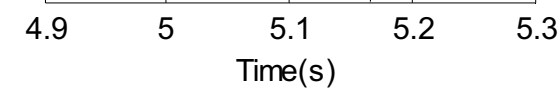

(b)
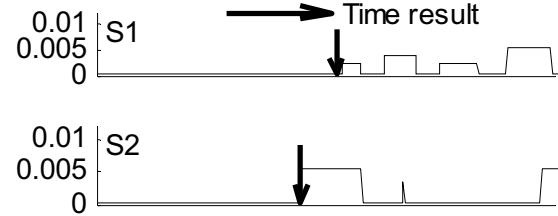

0.01 เ 3

0.005

0

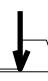

0.01
0.005
0
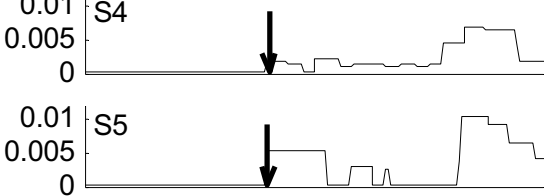

0.01 -

0.005

0

$5.1 \quad 5.11$

$\begin{array}{lll}5.12 & 5.13 & 5.14\end{array}$

Time(s)

(c) Original Amplitude Amplitude by WT


Fig. 4. First-arrival identification for six microseismic signals by the two automatic methods: (a) time series of six original microseismic signals, (b) period information used by the proposed method near the picking results, and (c) amplitude information used by the proposed method and the normalized reconstructed signals used by the WT method compared with the original signals near the picking results. 
Picking results of the microseismic signals

\begin{tabular}{|c|c|c|c|c|r|}
\hline \multirow{2}{*}{$\begin{array}{c}\text { Signal } \\
\text { number }\end{array}$} & \multirow{2}{*}{$\begin{array}{c}\text { Manual } \\
\text { results }\end{array}$} & \multicolumn{2}{|c|}{ Proposed method } & \multicolumn{2}{c|}{ WT method } \\
\cline { 3 - 6 } & Results & Error* & Results & \multicolumn{1}{|c|}{ Error* } \\
\hline 1 & 5.1250 & 5.1262 & 0.0008 & 5.1322 & 0.0072 \\
2 & 5.1220 & 5.1224 & 0.0004 & 5.1216 & -0.0004 \\
3 & 5.1210 & 5.1224 & 0.0014 & 5.1172 & -0.0038 \\
4 & 5.1200 & 5.1202 & 0.0002 & 5.1352 & 0.0152 \\
5 & 5.1230 & 5.1200 & -0.0030 & 5.1228 & -0.0002 \\
6 & 5.1190 & 5.1244 & 0.0054 & 5.1168 & -0.0022 \\
\hline
\end{tabular}

${ }^{*}$ The error means the difference between the manual picking results and those of the automated methods.

signals showed excellent accuracy and were smoother, especially for the first wave arrival. The main reason for the bigger picking errors of this method is the criterion for the first-pulse identification. For signals in the wide main frequency bands, only amplitude information can be used for the detection, and parameters such as the window length and thresholds which affect the accuracy of the picking results are difficult to determine, especially for low SNR signals. For low SNR signals, there are obvious noise wave in the denoised reconstructed signals, which may lead to incorrect picking results. The picking results of the WT method depend mostly on the LTA threshold; thus, when the time window or the thresholds change, the results will change accordingly.

For the ITD method, the denoised signals preserved the sudden change of the amplitude at the first-arrival, as well as extracting the period information consistent with the instantaneous amplitude. These two features make it easy to establish the evaluation criterion for the first-arrival identification. For every point, using the instantaneous piece-wise constant amplitude equal to the extremum value of the instantaneous half-wave that the point belongs to instead of the point's transient amplitude, makes the amplitude threshold easier to preset. The instantaneous equivalent period of the original signals was used not only for the evaluation criterion, but also for determining the location of the consecutive wave; therefore, the time windows can be adaptively determined. The thresholds of the proposed method were estimated based on the reference values from the period information and the picking time results were stable. These are the main advantages of the proposed ITD method, suggesting that in most cases the results of the proposed method are more reliable and stable than those of the WT method. 


\section{CONCLUSIONS}

Using the time-frequency-energy denoised amplitude signals and the filtered equivalent period (frequency), we present a method for detecting first-arrival signals and apply it to simulated signals and manmade blasting signals of high SNR and microseismic signals of low SNR for automatic first-arrival detecting.

The instantaneous amplitude and equivalent period information for the first-arrival identification were obtained by the proposed method using several decomposed component levels. In general, the first decomposed component of level 1 has some effect on the long-term-average amplitude and the average period for the first pulse identification in some cases; the second and third components are effective signals - the two maximum energy levels that contain the dominant frequency bands. The amplitude information uses the sum of the first several instantaneous amplitude components which should include the two maximum energy levels. The equivalent period information uses the energy weighted sum of the period components of the two maximum energy levels weighted by their average amplitude ratio.

The first pulse identification depends on four amplitude thresholds and period thresholds, including the instantaneous amplitude ratio and instantaneous period, and the average amplitude ratio and average period of the next waves connected with the detected point. The denoised piece-wise constant amplitude is used for each point to detect the first arrivals; the abrupt amplitude at the beginning of the first pulse makes the amplitude threshold easy to preset. The period thresholds were estimated based on the extracted period information. According to the equivalent period information, the adaptive time window was obtained for long-time average amplitude calculation.

When the sampling frequency is low, the abrupt amplitude information at the beginning of the first arrival pulse is retained in the proposed method; for the WT method, because of the inherent characteristics of the wavelet, there exists a distortion and phase shift phenomenon at the beginning of the first arrival in the denoised signals. Thus, the picking accuracy of the proposed method is less influenced by the sampling frequency of the original signals compared with that of the WT method.

Compared with the WT method, in most cases, the picking results of the proposed method were stable, more effective, and more reliable. The deviations between the results of the proposed method and the manual results are within $0.00 \pm 0.003 \mathrm{~s}$ in most cases, less than those of the WT method. The proposed method showed better performance in identifying $P$-arrivals of microseismic signals. 
Acknowledgments. This research was supported by the National Natural Science Foundation of China (Grant No. 51178185) and the Research Fund for the Program of Higher Education (Grant No. JG2013B03).

\section{References}

Allen, R. (1978), Automatic earthquake recognition and timing from single traces, Bull. Seismol. Soc. Am. 68, 5, 1521-1532.

Allen, R. (1982), Automatic phase pickers: Their present use and future prospects, Bull. Seismol. Soc. Am. 72, 6B, 225-242.

Baer, M., and U. Kradolfer (1987), An automatic phase picker for local and teleseismic events, Bull. Seismol. Soc. Am. 77, 4, 1437-1445.

Bai, C., and B.L.N. Kennett (2000), Automatic phase-detection and identification by full use of a single three-component broadband seismogram, Bull. Seismol. Soc. Am. 90, 1, 187-198, DOI: 10.1785/0119990070.

Dai, H., and C. MacBeth (1995), Automatic picking of seismic arrivals in local earthquake data using artificial neural network, Geophys. J. Int. 120, 3, 758-774, DOI: 10.1111/j.1365-246X.1995.tb01851.x.

Daubechies, I. (1992), Ten Lectures on Wavelets, SIAM, Philadelphia, 357 pp.

Earle, P.S., and P.M. Shearer (1994), Characterization of global seismograms using an automatic-picking algorithm, Bull. Seismol. Soc. Am. 84, 2, 366-376.

Frei, M.G., and I. Osorio (2007), Intrinsic time-scale decomposition: timefrequency-energy analysis and real-time filtering of non-stationary signals, Proc. R. Soc. A 463, 321-342, DOI: 10.1098/rspa.2006.1761.

Galiana-Merino, J.J., J. Rosa-Herranz, J. Giner, S. Molina, and F. Botella (2003), De-noising of short-period seismograms by wavelet packet transform, Bull. Seismol. Soc. Am. 93, 6, 2554-2562, DOI: 10.1785/0120010133.

Galiana-Merino, J.J., J. Rosa-Herranz, P. Jáuregui, S. Molina, and J. Giner (2007), Wavelet transform methods for azimuth estimation in local threecomponent seismograms, Bull. Seismol. Soc. Am. 97, 3, 793-803, DOI: $10.1785 / 0120050225$.

Liu, J.S., Y. Wang, and Z.X. Yao (2013), On micro-seismic first arrival identification: A case study, Chin. J. Geophys. 56, 5, 1660-1666, DOI: 10.6038/ cjg20130523.

Ma, Q. (2008), Study and application on earthquake early warning, Ph.D. Thesis, Institute of Engineering Mechanics, China Earthquake Administration, Harbin, China (in Chinese).

McEvilly, T.V., and E.L. Majer (1982), ASP: An Automated Seismic Processor for microearthquake networks, Bull. Seismol. Soc. Am. 72, 1, 303-325. 
Ye, G.X., F.X. Jiang, and S.H. Yang (2008), Possibility of automatically picking first arrival of microseismic wave by energy eigenvalue method, Chin. J. Geophys. 51, 5, 1574-1581.

Zhang, H., C. Thurber, and C. Rowe (2003), Automatic P-wave arrival detection and picking with multiscale wavelet analysis for single-component recordings, Bull. Seismol. Soc. Am. 93, 5, 1904-1912, DOI: 10.1785/0120020241.

Zhang, R.H., D.C. Lin, and L. Qiao (2011), Application of the optimum wavelet packet transform in de-noising seism signals, J. Seismol. Res. 34, 3, 358364.

Zhang, R.H., H.W. Qi, D.C. Lin, and L. Qiao (2012), Micro-seismic signal denoising based on intrinsic time-scale decomposition algorithm, Safety Coal Min. 43, 3, 164-168.

Received 25 January 2014 Received in revised form 26 November 2014 Accepted 9 December 2014 